\title{
Pre-implantation balloon-aortic valvuloplasty before transcatheter aortic valve implantation: is this still needed?
}

\author{
Rafail A. Kotronias ${ }^{1,2,3}$, Michael Teitelbaum ${ }^{4}$, Rodrigo Bagur ${ }^{3,4}$ \\ ${ }^{1}$ Oxford University Clinical Academic Graduate School, University of Oxford, Oxford, UK; ${ }^{2}$ Oxford Heart Centre, Oxford University Hospitals \\ NHS Foundation Trust, Oxford, UK; ${ }^{3}$ Keele Cardiovascular Research Group, Institute for Applied Clinical Science and Centre for Prognosis \\ Research, Institute of Primary Care and Health Sciences, University of Keele, Stoke-on-Trent, UK; ${ }^{4}$ London Health Sciences Centre, Western \\ University, London, Ontario, Canada \\ Contributions: (I) Conception and design: RA Kotronias, R Bagur; (II) Administrative support: R Bagur; (III) Provision of study materials or patients: \\ RA Kotronias, M Teitelbaum; (IV) Collection and assembly of data: RA Kotronias, R Bagur; (V) Data analysis and interpretation: RA Kotronias, R \\ Bagur; (VI) Manuscript writing: All authors; (VII) Final approval of manuscript: All authors. \\ Correspondence to: Rodrigo Bagur, MD, PhD, FAHA. University Hospital, London Health Sciences Centre, 339 Windermere Road, N6A 5A5, \\ London, Ontario, Canada. Email: rodrigobagur@yahoo.com.
}

\begin{abstract}
Balloon aortic valvuloplasty (BAV) has historically been recommended prior to transcatheter aortic valve implantation (TAVI). Pre-implantation BAV (pBAV) creates fractures at the level of calcified leaflets, thereby facilitating delivery of the transcatheter valve system across the diseased aortic valve and, enhances prosthesis implantation and expansion within the calcified aortic valve annulus. New device designs, lower profile delivery systems and increasing operator experience have enabled direct-TAVI (without pBAV), and its appeal amongst TAVI operators enhanced the dissemination of a direct TAVI approach across many centres. In this review, we discuss contemporary evidence that inform the debate on the need for routine pBAV for TAVI candidates and present a framework that may assist operators in selecting patients for pBAV.
\end{abstract}

Keywords: Transcatheter aortic valve implantation (TAVI); transcatheter aortic valve replacement (TAVR); balloon aortic valvuloplasty (BAV); direct implantation

Submitted Jun 11, 2018. Accepted for publication Jun 14, 2018.

doi: $10.21037 /$ jtd.2018.06.102

View this article at: http://dx.doi.org/10.21037/jtd.2018.06.102

\section{Introduction}

Balloon aortic valvuloplasty (BAV) has historically been recommended prior to transcatheter aortic valve implantation (TAVI). Pre-implantation BAV (pBAV) creates fractures at the level of calcified leaflets, thereby facilitating delivery of the transcatheter valve system across the diseased aortic valve and, enhances prosthesis implantation and expansion within the calcified aortic valve annulus. However, it has been hypothesised that pBAV may increase embolic events and confer to procedural hemodynamic instability.

New device designs, lower profile delivery systems (1) and increasing operator experience (2) have enabled directTAVI (without pBAV), and its appeal amongst TAVI operators enhanced the dissemination of a direct TAVI approach across many centres.

In this review, we discuss contemporary evidence that inform the debate on the need for routine pBAV for TAVI candidates and present a framework that may assist operators in selecting patients for $\mathrm{pBAV}$.

\section{Role of pBAV for valve system deliverability}

During the early stages of transfemoral TAVI, pBAV was mandatory to ensure the uneventful crossing of the high-profile first-generation valve systems through the stenosed valves. Mechanistically, it is plausible that forceful advancement of the delivery system and uncontrollable movements of the stiff wire in the ventricular cavity may 
lead to perforation and cardiac tamponade. However, bailout BAV has been reported for such instances with successful outcomes (3). Nonetheless, recently introduced lower-profile delivery systems (1) and increasingly experienced operators employing novel techniques such as partial valvuloplasty $(4,5)$ have limited the scope for routine pBAV in smoothly crossing the stenosed valve.

\section{Is pBAV required for optimal valve deployment?}

A meta-analysis of 16 single centre cohort studies including 1,395 patients suggested that a direct approach is safe in terms of numerous procedurally and clinically relevant endpoints when compared with pBAV (6). However, as acknowledged by the authors, the study pooled nonrandomised cohort studies and did not adjust for potential anatomical and clinical confounders owing to lack of patient-level data. Capturing the evolving paradigm shift, the UK and Israeli TAVI registries showed that pBAV in 2014 was performed in $\sim 60 \%$ of the TAVI caseload as opposed to $\sim 95 \%$ in $2008(2,7)$. In both studies, first generation SAPIEN (Edwards Lifesciences, Irvine, California, USA) and CoreValve (Medtronic, Inc., Minneapolis, Minnesota, USA) devices were predominantly investigated, though subgroup analyses did not identify significant interaction between valve type and outcome $(2,6)$. Indeed, device success rates $>96 \%$ have been reported with direct implantation of new generation devices such as SAPIEN-3 $(8,9)$ and the mechanically expanding Lotus Valve (Boston Scientific, Natick, Massachusetts, USA) $(10,11)$.

Clinically significant $(\geq 2+)$ paravalvular leakage (PVL) is associated to short- and long-term mortality following TAVI (12-14). As a result, post-implantation balloon dilatation is performed to immediately correct significant PVLs, however, with the inherent risks of device migration or annulus rupture $(15,16)$, and the unknown impact on long-term valve durability $(17,18)$. Recent studies have shown that moderate/severe PVL and post-implantation balloon dilatation are not significantly different between a direct-TAVI and pBAV approach, regardless of the TAVI device $(2,6)$. However, key predictors of PVL, namely extent of aortic valve calcification and calcium distribution were not available (19-21). Indeed, unfavourable features for direct-TAVI include: heavy or severe aortic valve calcification defined as leaflet thickness $>5 \mathrm{~mm}$, large nodules and diffuse calcification of the aortic annulus, an asymmetric and bulky calcification distribution, valve area
$<0.4 \mathrm{~cm}^{2}$ with an eccentric and/or irregular orifice, presence of calcification nodules at the left ventricle outflow tract or close to coronary ostia $(4,22,23)$.

Deployment optimisation is dependent on numerous factors such as operator, system utilized and patient. With increasing operator experience and new TAVI devices with high success rates and considerably reduced moderate/ severe PVL rates $(<5 \%)(24,25)$, the role of pBAV is becoming less prominent. The incorporation of routine multidetector computed tomography (MDCT) in TAVI pre-planning certainly helps identifying patients whose anatomy is hostile toward a direct implantation.

\section{Adjunctive utilities of pBAV}

\section{$B A V$ for transcatheter valve sizing and device selection in complex cases}

pBAV may be used to complement MDCT for transcatheter valve sizing and type of device selection in selected cases. For example, in cases where valve sizing based on MDCT falls between valve sizes, pBAV with concomitant supra-annular aortography may guide appropriate size selection (26). In addition, pBAV may also help determine the interaction of the prosthesis and coronary ostia in certain cases. Indeed, in patients with high risk features for coronary obstruction on MDCT; narrow, tubular aorta with low coronary ostia, pBAV with aortography can evaluate the interaction of the leaflet with the coronary ostium (27). Such information enables defaulting to a procedural strategy that firstly minimises risk for coronary obstruction, as well as implementing preventive measurements for bailout procedures such as guidewire engagement of the ostium at risk should urgent stenting be required (28), or simply choosing certain type of valve system that does not jail the coronary ostia (29-31).

\section{What are the risks of pBAV?}

\section{Hemodynamic instability}

pBAV can confer to hemodynamic compromise in a subset of patients owing to the temporary interruption in ventricular output during rapid pacing and/or new severe aortic regurgitation. Patients with any combination of pre-existent low flow-low gradient aortic stenosis, severe aortic regurgitation, severe left ventricular systolic dysfunction or severe pulmonary hypertension are at high risk of acute decompensation (32). Often, hemodynamic 
deterioration is sudden and profound and may lead to clinically significant multi-organ hypoperfusion. Therefore, careful consideration of MDCT parameters in patients with high-risk features for hemodynamic compromise is key to choosing a direct over a pBAV approach. High risk patients with hostile anatomies mandating pBAV may benefit from the use of novel valvuloplasty balloons $\left(\right.$ TRUE $^{\circledR}$ Flow Valvuloplasty Perfusion Catheter, Bard Peripheral Vascular, Inc. Tempe, Arizona, USA) that enable perfusion during pBAV inflation.

\section{Risk of systemic embolization}

Mechanistically, pBAV involves additional manipulations of the diseased aortic valve, hence, increasing the risk of aortic wall and calcific valve tissue emboli. Indeed, arterial wall, native valve tissue and calcific emboli are frequently identified in histological analyses of debris from cerebral embolic protection devices $(33,34)$ in TAVI patients. Furthermore, studies investigating the relation of pBAV in TAVI patients to the incidence and number of new emboli as assessed by cerebral diffusion-weighted magnetic resonance imaging found no significant difference when compared to a direct-TAVI approach (23). In this regard, Bijuklic et al. (23) showed that direct-TAVI was linked to a higher volume of ischemic lesions, speculating that pBAVinduced microfractures of the native calcified valve reduce the volume of embolised material.

Stroke event rates in patients with pBAV and directTAVI with first generation devices are similar; varying between $2 \%$ to $4 \%$ (2), and independent of the implanted system. Comparable event rates $(\sim 3 \%)$ are also seen with new generation devices whether they employ pBAV or not $(10,11)$. Indeed, standalone BAV has a $2 \%$ risk of stroke which was not significantly different to the stroke rate of TAVI patients (3.1\%) in a propensity matched analysis (35). In the absence of appropriately powered studies and compelling data to establish an undisputed link between pBAV, cerebral emboli and stroke in TAVR patients, inferences on pBAV and stroke ought to be interpreted cautiously. Nonetheless, patients harbouring high risk features for peri-procedural cerebrovascular accidents (36), may benefit from a tailored approach to valve deployment and embolic protection device utilisation.

\section{Shifting towards a tailored approach to pBAV}

Contemporary literature suggests that direct-TAVI in an appropriate subset of patients, is safe and yields comparable clinical outcomes when compared to a routine pBAV strategy. However, one should bear in mind that available data on the field emanate from observational studies with their inherent shortcomings. Despite the trend of increasing direct implantations, mostly using a balloon-expandable valve, there is no concerted approach to patient selection for direct-TAVI. Due to expansion of TAVI to low risk patients, attention is shifting on improving outcomes and healthcare resource utilisation. Consequently, operators will be responsible to case by case tailor their approach, ensuring that additional procedural steps are justified. To this avail, the results of the DIRECT (The preDIlatation in tRanscathEter aortiC Valve implanTation Trial, ClinicalTrials.gov: NCT02448927) and DIRECTAVI (TAVI Without Balloon Predilatation SAPIEN-3, ClinicalTrials. gov: NCT02729519) randomised controlled trials are anticipated. Both studies investigate the efficacy and safety of a direct as opposed to a pBAV strategy in CoreValve/ Evolut R and SAPIEN-3 system implantations respectively.

\section{Conclusions}

Routine pBAV in TAVI is declining as new devices and increasing operator experience enable optimisation of the procedural workflow. Contemporary data support the safety of a direct-TAVI approach; however, pBAV remains an important procedural stage during TAVI and further research is required to identify the subset of patients that may benefit from pBAV. Ultimately, it is up to the Heart Team to decide the best strategy based on anatomical characteristics and personal experience.

\section{Acknowledgements}

None.

\section{Footnote}

Conflicts of Interest: The authors have no conflicts of interest to declare.

\section{References}

1. Jones BM, Krishnaswamy A, Tuzcu EM, et al. Matching patients with the ever-expanding range of TAVI devices. Nat Rev Cardiol 2017;14:615.

2. Martin GP, Sperrin M, Bagur R, et al. Pre-Implantation 
Balloon Aortic Valvuloplasty and Clinical Outcomes Following Transcatheter Aortic Valve Implantation: A Propensity Score Analysis of the UK Registry. J Am Heart Assoc 2017;6.

3. Chan PH, Mario CD, Moat N. Transcatheter aortic valve implantation without balloon predilatation: not always feasible. Catheter Cardiovasc Interv 2013;82:328-32.

4. Abramowitz Y, Jilaihawi H, Chakravarty T, et al. Sapien 3 Transcatheter Aortic Valve Implantation With Moderate or Without Predilation. J Invasive Cardiol 2016;28:421-6.

5. Davies WR, Bapat VN, Hancock JE, et al. Direct TAVI using a balloon-expandable system: a novel technique to eliminate pre-deployment balloon aortic valvuloplasty. EuroIntervention 2014;10:248-52.

6. Bagur R, Kwok CS, Nombela-Franco L, et al. Transcatheter Aortic Valve Implantation With or Without Preimplantation Balloon Aortic Valvuloplasty: A Systematic Review and Meta-Analysis. J Am Heart Assoc 2016;5. doi: 10.1161/JAHA.115.003191.

7. Fink N, Segev A, Kornowski R, et al. Balloon dilatation and outcome among patients undergoing trans-femoral aortic valve replacement. Int J Cardiol 2017;230:537-41.

8. Bonaros N, Kofler M, Frank D, et al. Balloon-expandable transaortic transcatheter aortic valve implantation with or without predilation. J Thorac Cardiovasc Surg 2018;155:915-23.

9. Strauch J, Wendt D, Diegeler A, et al. Balloonexpandable transapical transcatheter aortic valve implantation with or without predilation of the aortic valve: results of a multicentre registry. Eur J Cardiothorac Surg 2018;53:771-7.

10. Ruparelia N, Thomas K, Newton JD, et al. Procedural and thirty-day outcomes following transfemoral implantation of the fully repositionable and retrievable Lotus valve without routine pre-dilatation in a consecutive patient cohort: a single-center experience. Cardiovasc Revasc Med 2018;19:78-82.

11. Tarantini G, Nai Fovino L, Tellaroli P, et al. TAVR with mechanically expandable prostheses: Is balloon aortic valvuloplasty really necessary? Int J Cardiol 2017;246:37-40.

12. Tamburino C, Capodanno D, Ramondo A, et al. Incidence and predictors of early and late mortality after transcatheter aortic valve implantation in 663 patients with severe aortic stenosis. Circulation 2011;123:299-308.

13. Moat NE, Ludman P, de Belder MA, et al. Long-term outcomes after transcatheter aortic valve implantation in high-risk patients with severe aortic stenosis: the U.K.
TAVI (United Kingdom Transcatheter Aortic Valve Implantation) Registry. J Am Coll Cardiol 2011;58:2130-8.

14. Abdel-Wahab M, Zahn R, Horack M, et al. Aortic regurgitation after transcatheter aortic valve implantation: incidence and early outcome. Results from the German transcatheter aortic valve interventions registry. Heart 2011;97:899-906.

15. Fiorina C, Maffeo D, Curello S, et al. Direct transcatheter aortic valve implantation with self-expandable bioprosthesis: feasibility and safety. Cardiovasc Revasc Med 2014;15:200-3.

16. Wang N, Lal S. Post-dilation in transcatheter aortic valve replacement: A systematic review and meta-analysis. J Interv Cardiol 2017;30:204-11.

17. Bagur R, Pibarot P, Otto CM. Importance of the valve durability-life expectancy ratio in selection of a prosthetic aortic valve. Heart 2017;103:1756-9.

18. Foroutan F, Guyatt GH, Otto CM, et al. Structural valve deterioration after transcatheter aortic valve implantation. Heart 2017;103:1899-905.

19. Buellesfeld L, Stortecky S, Heg D, et al. Extent and distribution of calcification of both the aortic annulus and the left ventricular outflow tract predict aortic regurgitation after transcatheter aortic valve replacement. EuroIntervention 2014;10:732-8.

20. Ewe SH, Ng AC, Schuijf JD, et al. Location and severity of aortic valve calcium and implications for aortic regurgitation after transcatheter aortic valve implantation. Am J Cardiol 2011;108:1470-7.

21. Khalique OK, Hahn RT, Gada H, et al. Quantity and Location of Aortic Valve Complex Calcification Predicts Severity and Location of Paravalvular Regurgitation and Frequency of Post-Dilation After Balloon-Expandable Transcatheter Aortic Valve Replacement. JACC Cardiovasc Interv 2014;7:885-94.

22. Islas F, Almeria C, Garcia-Fernandez E, et al. Usefulness of echocardiographic criteria for transcatheter aortic valve implantation without balloon predilation: a single-center experience. J Am Soc Echocardiogr 2015;28:423-9.

23. Bijuklic K, Haselbach T, Witt J, et al. Increased Risk of Cerebral Embolization After Implantation of a Balloon-Expandable Aortic Valve Without Prior Balloon Valvuloplasty. JACC Cardiovasc Interv 2015;8:1608-13.

24. Popma JJ, Reardon MJ, Khabbaz K, et al. Early Clinical Outcomes After Transcatheter Aortic Valve Replacement Using a Novel Self-Expanding Bioprosthesis in Patients With Severe Aortic Stenosis Who Are Suboptimal for Surgery. JACC Cardiovasc Interv 2017;10:268-75. 
25. Ben-Shoshan J, Konigstein M, Zahler D, et al. Comparison of the Edwards SAPIEN S3 Versus Medtronic Evolut-R Devices for Transcatheter Aortic Valve Implantation. Am J Cardiol 2017;119:302-7.

26. Otto CM, Kumbhani DJ, Alexander KP, et al. 2017 ACC Expert Consensus Decision Pathway for Transcatheter Aortic Valve Replacement in the Management of Adults With Aortic Stenosis: A Report of the American College of Cardiology Task Force on Clinical Expert Consensus Documents. J Am Coll Cardiol 2017;69:1313-46.

27. Bagur R, Dumont E, Doyle D, et al. Coronary Ostia Stenosis After Transcatheter Aortic Valve Implantation. JACC Cardiovasc Interv 2010;3:253-5.

28. Chakravarty T, Sharma R, Abramowitz Y, et al. Outcomes in Patients With Transcatheter Aortic Valve Replacement and Left Main Stenting: The TAVR-LM Registry. J Am Coll Cardiol 2016;67:951-60.

29. Bagur R, Teefy PJ, Kiaii B, et al. First North American experience with the transfemoral ACURATE-neo(TM) self-expanding transcatheter aortic bioprosthesis. Catheter Cardiovasc Interv 2017;90:130-8.

30. Bagur R, Pestrichella V, Montesanti R, et al. Transfemoral transcatheter ACURATE-neo aortic valve replacement in a patient with a previous mechanical mitral valve. J Card Surg 2017;32:358-60.

31. Kotronias RA, Mamas MA, Bagur R. Revascularizing coronary artery disease in patients undergoing transcatheter aortic valve implantation. J Thorac Dis 2018;10:E79-82.

32. Suh WM, Witzke CF, Palacios IF. Suicide left ventricle following transcatheter aortic valve implantation. Catheter Cardiovasc Interv 2010;76:616-20.

33. Kapadia SR, Kodali S, Makkar R, et al. Protection Against Cerebral Embolism During Transcatheter Aortic Valve Replacement. J Am Coll Cardiol 2017;69:367-77.

34. Bagur R, Solo K, Alghofaili S, et al. Cerebral Embolic Protection Devices During Transcatheter Aortic Valve Implantation: Systematic Review and Meta-Analysis. Stroke 2017;48:1306-15.

35. Alkhouli M, Zack CJ, Sarraf M, et al. Morbidity and Mortality Associated With Balloon Aortic Valvuloplasty: A National Perspective. Circ Cardiovasc Interv 2017;10.

36. Mastoris I, Schoos MM, Dangas GD, et al. Stroke after transcatheter aortic valve replacement: incidence, risk factors, prognosis, and preventive strategies. Clin Cardiol 2014;37:756-64.
Cite this article as: Kotronias RA, Teitelbaum M, Bagur R. Pre-implantation balloon-aortic valvuloplasty before transcatheter aortic valve implantation: is this still needed? J Thorac Dis 2018;10(Suppl 30):S3599-S3603. doi: 10.21037/ jtd.2018.06.102 